\title{
Chemoresistance-associated alternative splicing signatures in serous ovarian cancer
}

\author{
TIANSHUI SUN and QING YANG \\ Department of Obstetrics and Gynecology, Shengjing Hospital of China Medical University, \\ Shenyang, Liaoning 110004, P.R. China
}

Received July 15, 2019; Accepted February 21, 2020

DOI: $10.3892 / \mathrm{ol} .2020 .11562$

\begin{abstract}
Primary platinum-based chemoresistance occurs in $\sim 30 \%$ of patients with serous ovarian cancer. Chemoresistance is the main cause of disease recurrence, and accurate predictors to identify these patients with chemoresistance are required. Alternative splicing (AS) is a post-transcriptional modification process that is altered in cancer. A possible association between AS and chemoresistance is unclear and needs to be studied comprehensively in ovarian cancer. In the present study, RNA-sequencing data and clinical information for 320 patients with ovarian serous cystadenocarcinoma (OV) were downloaded from The Cancer Genome Atlas (TCGA) database. Splicing events were determined using the TCGA SpliceSeq tool. Seven types of AS events were identified. Univariate and multivariate logistic analyses were performed, and predictive models for OV chemoresistance were established, as well as a splicing network. A total of 22,036 AS events were identified in 7,404 genes, with 915 AS events detected in 677 genes that were significantly associated with chemoresistance in patients with OV. A receiver operating characteristic (ROC) curve was constructed for resistance predictive models composed of the most significant AS events. The area under the ROC curve was 0.931, indicating strong and efficient prediction of chemoresistance. Additionally, the high-risk score was associated with shorter overall survival. The splicing correlation network suggested a potential role of splicing factors in chemoresistance. In summary, the present study created a powerful predictor for primary platinum-based chemoresistance in patients with OV, identified splicing networks that could be involved in potential mechanisms of chemoresistance and provided potential targets to overcome chemoresistance.
\end{abstract}

Correspondence to: Professor Qing Yang, Department of Obstetrics and Gynecology, Shengjing Hospital of China Medical University, 36 Sanhao Street, Heping, Shenyang, Liaoning 110004, P.R. China

E-mail: yangqing_sj@126.com

Key words: ovarian cancer, alternative splicing, chemotherapy resistance, The Cancer Genome Atlas, splicing factor

\section{Introduction}

Ovarian cancer was the fifth-leading cause of cancer-associated mortality in females in 2015 in the United States, with 295,414 newly-diagnosed cases globally in 2018 and 184,799 cancer-associated deaths globally in $2018(1,2)$. Statistical analyses indicate that $90 \%$ of ovarian cancer cases are epithelial, with serous carcinoma being the most common pathological type with a 5-year survival rate of $43 \%$ (3). Conventional treatment for epithelial ovarian cancer involves cytoreductive surgery followed by platinum- and taxane-based chemotherapy (4). However, development of resistance to chemotherapy eventually induces recurrence after treatment (5). An accurate and robust predictive marker of chemoresistance is urgently required to improve individualized treatment and enhance the prognosis and survival of patients with epithelial ovarian cancer. Previous studies have identified a number of chemoresistance-associated biomarkers, such as reactive stroma signature, markers of cancer stem cells and miRNAs (6-10), but they have not been used in clinical practice. Effective predictors of primary platinum-based chemotherapy resistance would provide novel strategies for treating patients with epithelial ovarian cancer.

Dysregulation of genomic expression serves a critical role in tumorigenesis and chemoresistance in epithelial ovarian cancer. Previous progress in developing genomics-based and precision-targeted therapies has provided novel strategies for treating patients with ovarian cancer (11). However, previous studies have only focused on gene expression levels rather than investigating how alternative splicing (AS) can affect transcript architecture $(12,13)$.

AS is a post-transcriptional modification process that produces a variable mature mRNA transcript from a single gene by removing different intronic or exonic regions from the precursor mRNA and subsequently combining the spliced exons $(14,15)$. AS generates mRNAs with different stabilities or coding potentials, enabling quantitative control of protein production and achieving distinct protein functions (16). AS serves crucial roles in specialized muscle functions (17), angiogenesis (18) and pathological processes, including hearing loss (19), Huntington's disease (20) and cancer (21). Emerging evidence suggests that AS is associated with tumorigenic processes, such as tumor proliferation, invasion, metastasis and apoptosis (22). Splicing factors perform splicing by binding 
to pre-mRNAs, influencing exon selection and selecting the splicing site (23). Splicing factors are expressed differentially between normal and cancerous tissues $(24,25)$. Therefore, identifying AS signature profiles and exploring splicing factors may reveal useful cancer biomarkers.

An analysis of AS in cancer has become possible with the advent of deep-sequencing techniques that allow the discovery of previously unknown prognostic and therapeutic biomarkers for patients with cancer. Prognostic predictors based on AS events have been identified in patients with various types of cancer, including ovarian cancer (26-28). However, to the best of our knowledge, no systematic analyses of chemoresistance-associated AS in ovarian cancer have been performed, even though these are urgently required due to the major role of chemoresistance in disease recurrence. In the present study, The Cancer Genome Atlas (TCGA) RNA-sequencing (RNA-seq) data was used to investigate whether AS events could serve as predictors of primary platinum-based chemotherapy resistance in serous ovarian carcinoma.

\section{Materials and methods}

Data acquisition. AS profiles were analyzed using the TCGA SpliceSeq tool version 1 provided by the MD Anderson Cancer Center (https://bioinformatics.mdanderson. org/TCGASpliceSeq/) (29). Seven types of AS events were quantified using the percent spliced-in (PSI) value: Exon skip (ES), alternate promoter (AP), alternate terminator (AT), alternative acceptor site (AA), alternate donor site (AD), retained intron (RI) and mutually exclusive exons (ME). The PSI values for the seven types of AS in ovarian serous cystadenoma (OV) were downloaded from TCGA SpliceSeq. AS events with a standard deviation $>0.05$ and a PSI value $>75 \%$ were included. Clinical information for the TCGA-OV cohort was obtained from the TCGA database (https://portal. gdc.cancer.gov/projects/TCGA-OV) (30). Individuals who met the following criteria were included in the present study: i) Patients diagnosed with serous ovarian cancer; ii) patients who received platinum-based chemotherapy; and iii) patients with well-defined responses to chemotherapy. Patients without AS information were excluded from the present study. A total of 63 splicing factors and their information were obtained from SpliceAid 2 (31). Level three mRNA expression data of splicing factors were also acquired from the TCGA database.

Statistical analysis. Univariate logistic regression analyses were performed to assess the predictive value of AS events for primary platinum-based chemotherapy resistance. Subsequently, the top 30 most significant AS events from the univariate analyses were included in multivariate logistic regression analyses to build prediction models for each type of AS event individually and for all types of AS events combined. The Akaike information criterion was applied to select the most appropriate risk model (32). The prediction accuracy of the risk models was evaluated by receiver operating characteristic (ROC) analysis. Patients were classified into high- and low-risk groups, with the median score as the cut-off value. Kaplan-Meier analysis and a log-rank test were performed to estimate the difference in overall survival (OS) time between the high- and low-risk groups.
Table I. Demographic and clinical characteristics of ovarian serous cystadenocarcinoma cases in The Cancer Genome Atlas datasets involved in developing alternative splicing signatures to predict primary platinum-based chemoresistance.

\begin{tabular}{lccr}
\hline Characteristics & $\begin{array}{c}\text { Resistance } \\
\text { cases, } \mathrm{n}\end{array}$ & $\begin{array}{c}\text { Sensitive } \\
\text { cases, } \mathrm{n}\end{array}$ & P-value \\
\hline Sample number & 95 & 225 & \\
Age, years & 56 & 128 & 0.734 \\
$<60$ & 39 & 97 & \\
$\geq 60$ & & & 0.027 \\
Stage & 1 & 16 & \\
FIGO I/II & 94 & 209 & \\
FIGO III/IV & & & \\
Grade & 12 & 26 & \\
Low & 81 & 194 & \\
High & 2 & 5 & \\
Unknown & & & \\
Debulking status & 53 & 164 & \\
Optimal & 36 & 25 & \\
Suboptimal & 6 & & \\
Unknown & & & \\
\end{tabular}

FIGO, International Federation of Gynecology and Obstetrics.

Resistance-associated splicing factor genes were identified using univariate logistic regression analysis. Pearson's correlation test was used to determine whether expression of the splicing factor genes was significantly associated with the PSI values of resistance-associated AS events. The regulatory network map was built based on the significantly correlated splicing factors and AS events.

All analyses were performed using $\mathrm{R}$ (version 3.5.2; www.r-project.org). $\mathrm{P}<0.05$ was considered to indicate a statistically significant difference, unless otherwise specified. Differences in clinicopathologic parameters between chemosensitive and chemoresistant groups, including age, grade, FIGO (International Federation of Gynecology and Obstetrics) stage and debulking status (33), were tested by unpaired t-test or the $\chi^{2}$ test.

Procedures. R was used to perform the univariate and multivariate logistic analyses and build chemoresistance prediction models. UpSet plots were generated using UpSetR (version 1.4.0; https://cran.r-project.org/web/packages/UpSetR/index.html).

The pROC package (version 1.13.0; https://cran.r-project. org/src/contrib/Archive/pROC/) was used to create ROC curves and to calculate the area under the curve (AUC). The Functional Annotation Result Summary tool version 6.8 (https://david.ncifcrf.gov/summary.jsp) from the Database for Annotation, Visualization, and Integrated Discovery (version 6.8) was used for Gene Ontology (GO) (http://geneontology.org) analysis of the corresponding genes (34). The gene interaction network and correlation network were visualized using Cytoscape (version 3.7.1; https://cytoscape.org). 
A 22,036 AS events of 7,404 genes in OV from TCGA SpliceSeq tool

Univariate logistic regression analysis

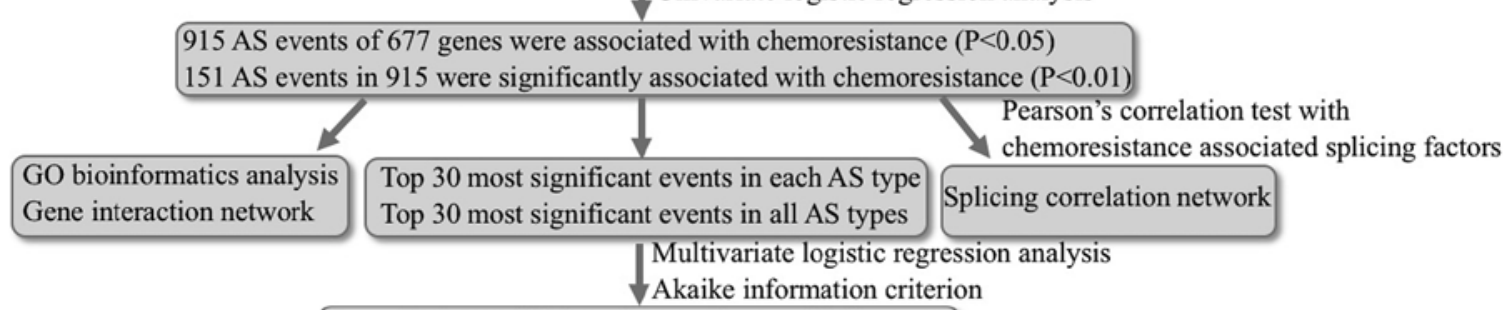

7 predictive models based on each type of AS events

1 predictive model based on all types of AS events

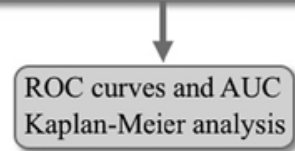

B
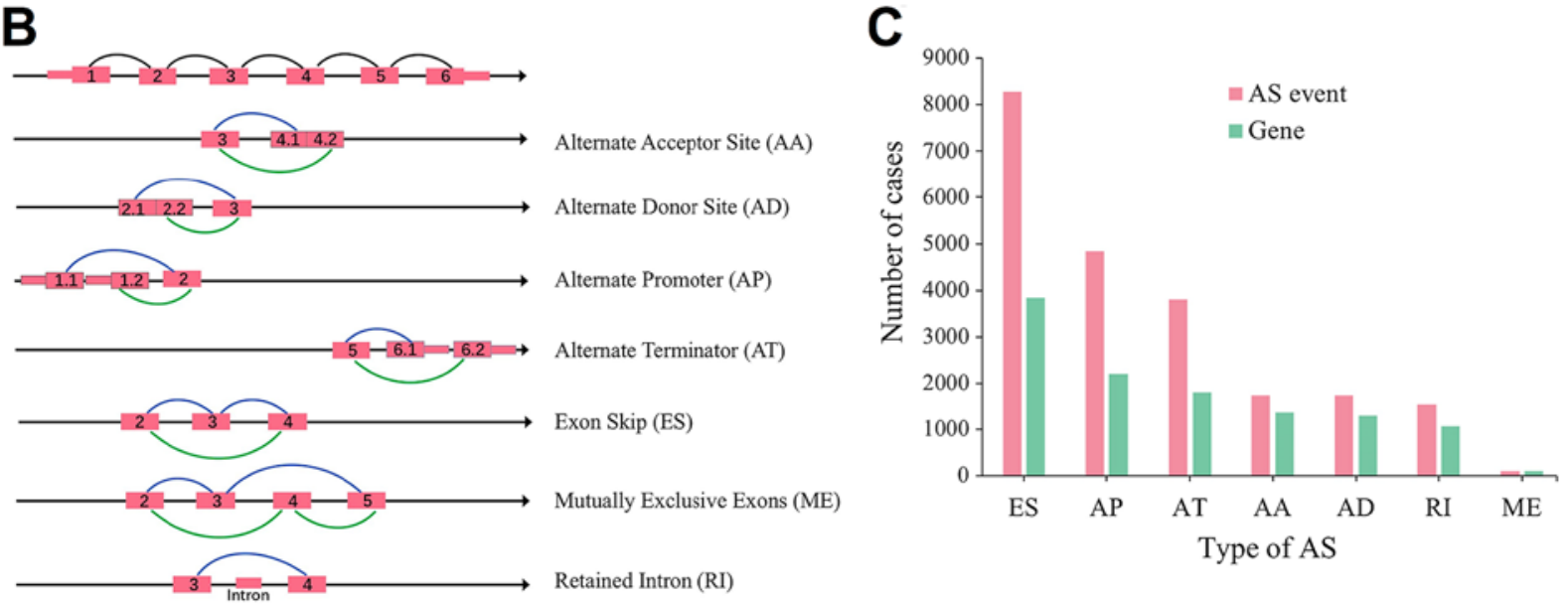

Figure 1. Overview of the seven types of AS. (A) Flowchart of the present study. (B) Illustrations of the seven types of AS events, including AA, AD, AP, AT, ES, ME and RI. (C) Number of AS events and involved genes from 320 patients with OV. AS, alternative splicing; OV, ovarian serous cystadenocarcinoma; AA, alternate acceptor site; AD, alternate donor site; AP, alternate promoter; AT, alternate terminator; ES, exon skip; ME, mutually exclusive exons; RI, retained intron; TCGA, The Cancer Genome Atlas; GO, Gene Ontology; ROC, receiver operating characteristic; AUC, area under the curve.

\section{Results}

Comprehensive analysis of AS events in the OV data. The overall process of the present study is described in Fig. 1A. Integrated AS event signatures for 320 patients with OV were curated from the TCGA database (Table I). Seven types of AS events were identified, as shown in Fig. 1B. A total of 22,036 AS events were detected in 7,404 genes, suggesting that one gene might have had more than one AS event. The following numbers of AS events were detected for each type: 8,280 ES events in 3,835 genes; $1,535 \mathrm{RI}$ events in 1,073 genes; 4,841 AP events in 2,196 genes; 3,806 AT events in 1,801 genes; 1,735 AD events in 1,291 genes; 1,741 AA events in 1,357 genes; and $98 \mathrm{ME}$ events in 96 genes (Fig. 1C). The most common type of AS events was ES, followed by AP and AT events.

Chemoresistance-associated AS events in the OV data. The univariate logistic regression analyses of OV data from the TCGA database identified 915 AS events associated with chemotherapy resistance in patients with $\mathrm{OV}(\mathrm{P}<0.05$; Table SI). Among these, 151 AS events were significantly associated with chemotherapy resistance ( $\mathrm{P}<0.01$; Table SII), 407
AS events were risk factors for chemotherapy resistance [odds ratio $(\mathrm{OR})>1$ ], and 508 were protective factors for chemotherapy resistance $(\mathrm{OR}<1)$. The distribution of 677 genes involved in 915 AS events was visualized in the UpSet plot (Fig. 2A). A total of 640 genes had only one type of AS event associated with chemoresistance, whereas 37 genes had more than one type of AS event associated with them. For example, ES, AA and AD events in GPR56 were all significantly associated with chemoresistance (Table SI).

GO bioinformatics analysis was performed on 677 genes with AS events. A total of 13 biological processes and 6 molecular functions were identified in the GO analysis $(\mathrm{P}<0.01$; Fig. 2B). These genes were found to be significantly associated with 'protein binding' and 'negative regulation of transcription from RNA polymerase II promoter'. The gene interaction network analysis for these 677 genes revealed a hub that included RHOA, POLR2G, RPS9, DYNLL1 and RPL13A (the top 5 genes with higher degree of connectivity) (Fig. 2C).

Chemoresistance predictors for patients with $O V$. The top 30 most significant events for each AS type (except for ME, which had only 6 events) and for all types of AS events were 
Table II. General characteristics of chemoresistance predictors for ovarian cancer.

\begin{tabular}{|c|c|c|}
\hline $\begin{array}{l}\text { Alternative } \\
\text { splicing }\end{array}$ & Formula & $\begin{array}{l}\text { OR }(95 \% \text { CI) } \\
\text { (High vs. low) }\end{array}$ \\
\hline AA & $\begin{array}{l}\text { 25.108023-4.442929*‘TSACC-8246-AA'+3.315118* } \\
\text { 'FAM111A-16027-AA'-5.747130*‘SERPINA1-29121-AA'-7.858303* } \\
\text { 'TNK1-38931-AA'-10.248344*'USHBP1-48249-AA'-11.439197* } \\
\text { 'DNAAF3-52039-AA'-11.843241*‘POLM-79455-AA' }\end{array}$ & $7.14(3.65-14.85)$ \\
\hline $\mathrm{AD}$ & 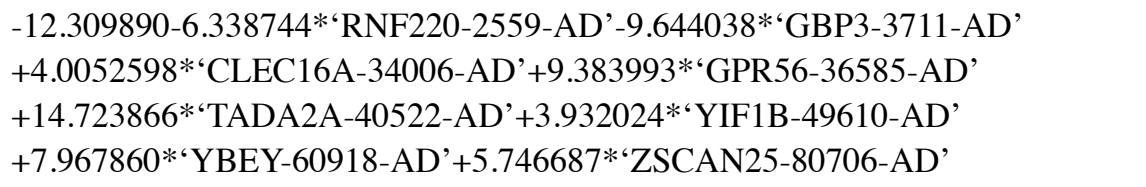 & $9.55(5.18-18.71)$ \\
\hline AP & $\begin{array}{l}\text { 8.898032-4.064766*'ANGEL2-9775-AP'-3.745283* } \\
\text { 'KIAA0391-27213-AP'+2.045282*`PPP1R13L-50435-AP'-9.158386* } \\
\text { 'FAM110A-58466-AP'-5.969009*`RBM47-69086-AP'-6.474537* } \\
\text { 'MYO10-71601-AP'-5.998058*`NUDT1-78608-AP'+2.415810* } \\
\text { 'MID1-88461-AP' }\end{array}$ & $6.68(4.06-11.41)$ \\
\hline AT & 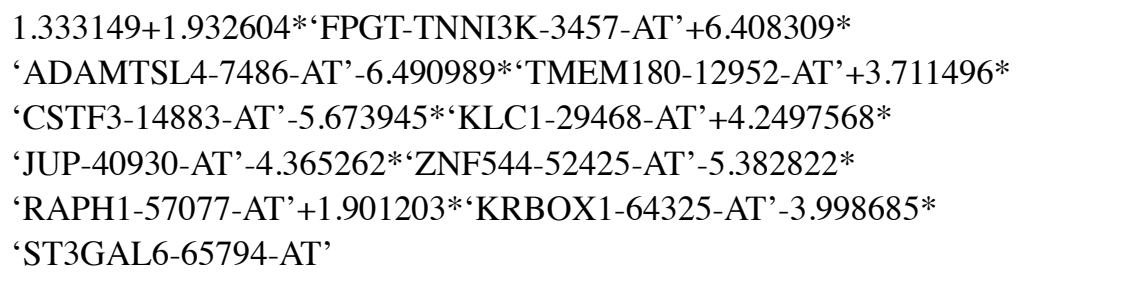 & $11.95(6.78-22.31)$ \\
\hline ES & $\begin{array}{l}\text { 8.884081+12.456874*`BTAF1-12524-ES'+3.040003*`SPAG9-42494-ES' } \\
+6.529384 *{ }^{*} \mathrm{GAA}-44021-\mathrm{ES} \text {-6.241932*`RBM6-64950-ES'-5.268264* } \\
\text { 'SLC10A7-70775-ES'+6.110066*`TRAPPC13-72245-ES'-6.730836* } \\
\text { 'PNISR-77056-ES'-4.439848*`PEX2-84241-ES'-5.337279* } \\
\text { 'EEF1D-98099-ES'-12.321102*`COL1A2-1412008-ES' }\end{array}$ & $22.05(10.31-54.59)$ \\
\hline ME & 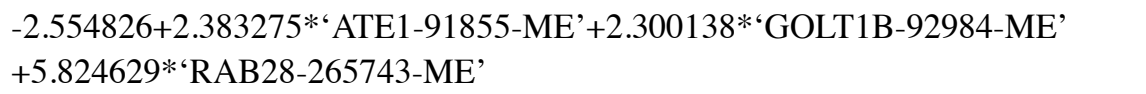 & $2.13(1.36-3.35)$ \\
\hline RI & 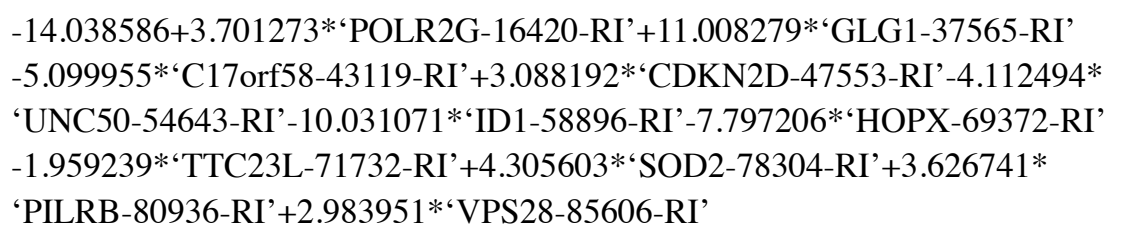 & $10.23(5.52-20.39)$ \\
\hline All & $\begin{array}{l}\text { 14.333930-8.697005*`SERPINA1-29121-AA'+5.713988*'SMIM7-48190-AD'- } \\
\text { 9.355225*'TRAPPC6B-27360-ES'+9.130642*`GAA-44021-ES'-2.458800* } \\
\text { 'PDE4D-72144-AP'-5.978179*`RBM6-64950-ES'-4.543145* } \\
\text { 'SLC10A7-70775-ES'+11.331974*`TRAPPC13-72245-ES'-5.850540* } \\
\text { 'FAM49B-85160-ES'-6.577849*`EEF1D-98099-ES'+5.275202* } \\
\text { 'CLEC16A-34006-AD'-11.452096*'COL1A2-1412008-ES'-8.721743* } \\
\text { 'ZNF544-52425-AT' }\end{array}$ & $64.88(22.55-284.86)$ \\
\hline
\end{tabular}

$\mathrm{OR}$, odds ratio; AA, alternate acceptor site; $\mathrm{AD}$, alternate donor site; $\mathrm{AP}$, alternate promoter; AT, alternate terminator; ES, exon skip; ME, mutually exclusive exons; RI, retained intron.

selected as candidates to identify the independent predictive model for chemoresistance in OV (Table SIII). Multivariate logistic regression analysis was performed for the 30 candidate events for each AS type and for all AS types combined, and the Akaike information criterion was used to select the most appropriate risk model (32). The predictive models are presented in Table II. The median score was used as the cut-off value, the patients were divided into high- and low-risk groups, and the OR for each model was calculated. ROC curves were generated and the AUCs were determined to evaluate the effectiveness of the chemoresistance predictive models. The seven predictors that were built using the seven types of AS events displayed considerable power in distinguishing the chemotherapy response of patients with OV. The model based on ES events was the most effective predictor among the models based on each type of AS event, with an AUC of 0.894 (Fig. 3). 

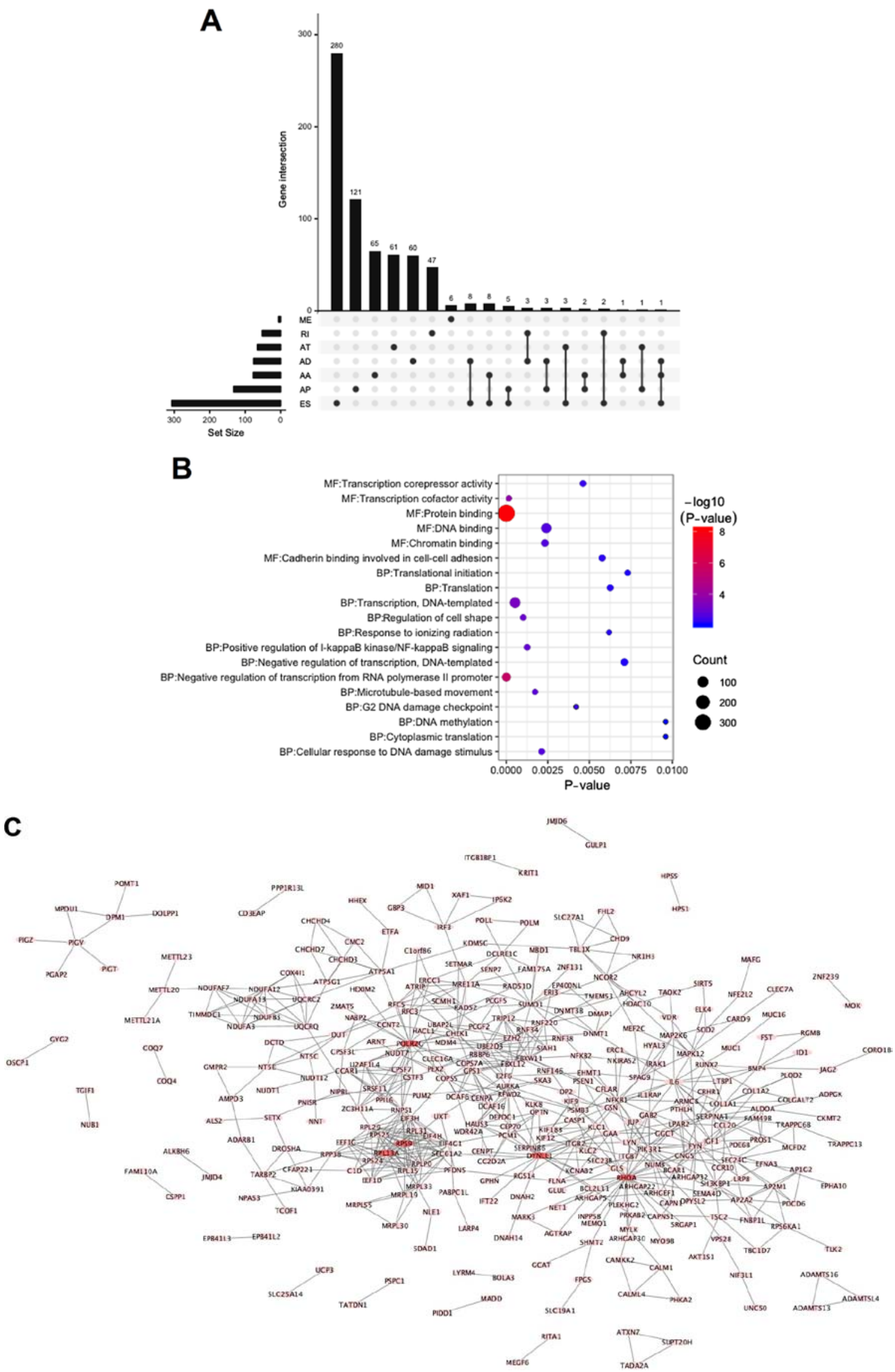

Figure 2. UpSet plot, GO analysis and gene network of chemoresistance-associated AS in OV. (A) UpSet plot of interactions between the seven types of chemoresistance-associated AS events in OV. (B) GO analysis of chemoresistance-associated AS events in OV. (C) Gene network of chemoresistance-associated AS in OV generated by Cytoscape. AS, alternative splicing; OV, ovarian serous cystadenocarcinoma; AA, alternate acceptor site; AD, alternate donor site; AP, alternate promoter; AT, alternate terminator; ES, exon skip; ME, mutually exclusive exons; RI, retained intron; GO, Gene Ontology; BP, biological process; MF, molecular function. 
Table III. Information for AS event candidates involved in the model based on all types of AS events.

\begin{tabular}{lcccc}
\hline Gene & OR $(95 \%$ CI $)$ & P-value & Type & Exon \\
\hline SMIM7 & $41.5291(8.0671-228.7526)$ & 0.00024 & AD & 7.2 \\
COL1A2 & $0.0043(0.0003-0.0549)$ & 0.00061 & ES & $23: 24: 25: 26: 27: 28: 29: 30: 31: 32: 33: 34: 35: 36: 37: 45: 46: 47$ \\
GAA & $130.2634(13.7266-1,594.0712)$ & 0.00075 & ES & 2.2 \\
EEF1D & $0.0247(0.0035-0.1586)$ & 0.00133 & ES & 6 \\
ZNF544 & $0.0508(0.0107-0.2331)$ & 0.00142 & AT & 10.2 \\
SLC10A7 & $0.0916(0.0257-0.3090)$ & 0.00151 & ES & 13 \\
FAM49B & $0.0373(0.0064-0.2078)$ & 0.00186 & ES & 5 \\
RBM6 & $0.0705(0.0163-0.2871)$ & 0.00233 & ES & $4: 05: 06$ \\
TRAPPC6B & $0.0109(0.0009-0.1213)$ & 0.00235 & ES & 11.2 \\
CLEC16A & $10.9329(3.0549-40.9032)$ & 0.00237 & AD & 1 \\
PDE4D & $0.3015(0.1554-0.5813)$ & 0.00274 & AP & 9 \\
TRAPPC13 & $25.9213(4.4025-167.8280)$ & 0.00322 & ES & 2.4 \\
SERPINA1 & $0.03190(0.0043-0.2147)$ & 0.00357 & AA &
\end{tabular}

OR, odds ratio; AA, alternate acceptor site; AD, alternate donor site; AP, alternate promoter; AT, alternate terminator; ES, exon skip; AS, alternative splicing.

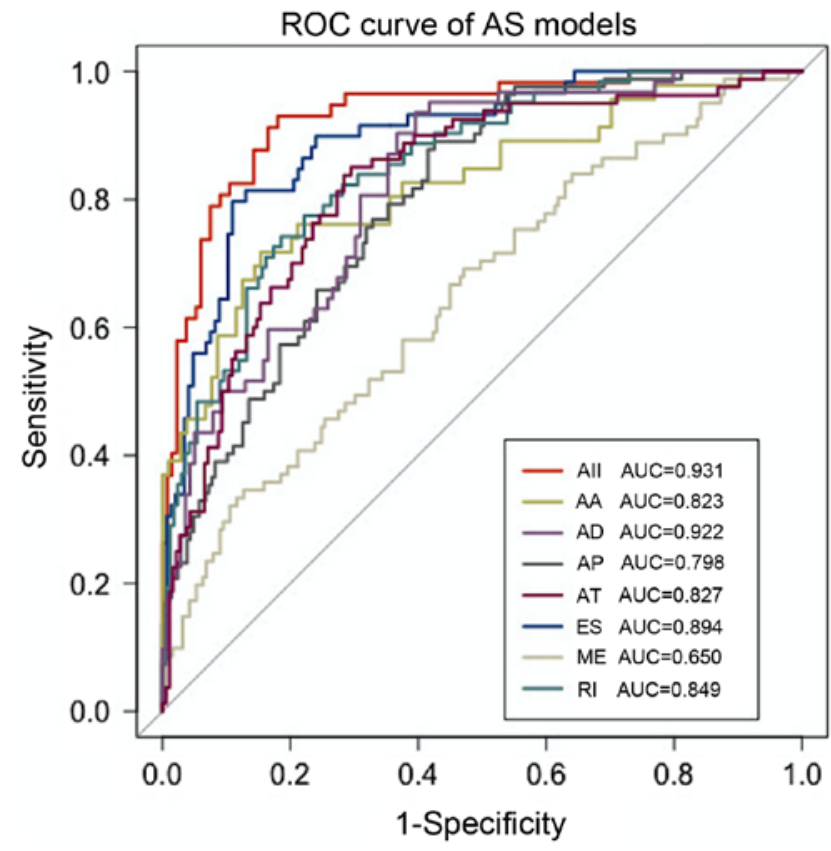

Figure 3. ROC curves with AUCs of chemoresistance predictors built by one type or all seven types of AS events in ovarian serous cystadenocarcinoma. AS, alternative splicing; ROC, receiver operating characteristic; AUC, area under the curve; AA, alternate acceptor site; AD, alternate donor site; AP, alternate promoter; AT, alternate terminator; ES, exon skip; ME, mutually exclusive exons; RI, retained intron.

The model based on all types of AS events exhibited the best efficiency with an AUC of 0.931. The information for AS event candidates involved in this model is presented in Table III. This model was utilized in univariate and multivariate logistic analyses of chemotherapy resistance together with common clinical characteristics. A high-risk score was an independent risk factor for chemoresistance (Table IV).
To verify the prognostic value of these predictive models, Kaplan-Meier analysis and log-rank tests were performed for each model. The results indicated that the patients in the high-risk groups in risk models based on AP, ES, RI and all types of AS events had shorter survival time compared with patients in the low-risk groups (Fig. 4). In the risk model based on all types of AS events, the median OS time for the highand low-risk groups were 1,341 and 1,875 days, respectively (Fig. 4H).

Potential correlation network of AS splicing factors. AS is regulated primarily by splicing factors. Therefore, it is crucial to determine whether key splicing factors regulate chemoresistance-associated AS events in OV. Univariate logistic analyses revealed that the mRNA expression levels of five splicing factors were associated with chemoresistance. Information of these splicing factors was obtained from SpliceAid2 and was shown in Table V. Subsequently, correlation analyses of the expression levels of the five splicing factors and the PSI values of $151 \mathrm{AS}$ events were performed $(\mathrm{P}<0.01$ in univariate analyses). A splicing correlation network was generated from the significant correlations $(\mathrm{P}<0.05$; Fig. 5A) between 70 chemoresistance-associated AS events, including 38 protective and 32 adverse AS events, and the 5 splicing factors. Most of the protective AS events were positively correlated with the expression of splicing factors, such as AP PSI value of SH3YL1 with expression of PTBP1, AD PSI value of RPL15 with expression of YBX1, AP PSI value of CLUL1 with expression of SYNCRIP. Most of the adverse AS events were negatively correlated with the expression of splicing factors, such as AT PSI value of UBAP2L with expression of TRA2B, ES PSI value of RPS24 with expression of SYNCRIP, ES PSI value of RHOA with expression of ELAVL4. Representative correlations between AS events and splicing factors are shown in the dot plots (Fig. 5B-G). 
Table IV. Univariate and multivariate logistic regression analyses for chemoresistance in The Cancer Genome Atlas datasets.

\begin{tabular}{|c|c|c|c|c|c|c|}
\hline \multirow[b]{2}{*}{ Characteristics } & \multicolumn{2}{|c|}{ Univariate } & \multirow[b]{2}{*}{ P-value } & \multicolumn{2}{|c|}{ Multivariate } & \multirow[b]{2}{*}{ P-value } \\
\hline & OR & $95 \% \mathrm{CI}$ & & OR & $95 \% \mathrm{CI}$ & \\
\hline \multicolumn{7}{|l|}{ Age, years } \\
\hline$<60$ & 1 (reference) & & & 1 (reference) & & \\
\hline$\geq 60$ & 0.90 & $0.52-1.53$ & 0.741 & 1.72 & $0.77-3.91$ & 0.270 \\
\hline \multicolumn{7}{|l|}{ Stage } \\
\hline FIGO I/II & 1 (reference) & & & 1 (reference) & & \\
\hline FIGO III/IV & 4.10 & $0.94-41.14$ & 0.186 & $\inf$ & 0 -inf & 0.989 \\
\hline \multicolumn{7}{|l|}{ Grade } \\
\hline Low & 1 (reference) & & & 1 (reference) & & \\
\hline High & 0.99 & $0.43-2.42$ & 0.978 & 0.37 & $0.06-1.61$ & 0.290 \\
\hline \multicolumn{7}{|l|}{ Debulking status } \\
\hline Optimal & 1 (reference) & & & 1 (reference) & & \\
\hline Suboptimal & 2.53 & $1.38-4.63$ & 0.012 & 5.13 & $2.01-15.21$ & 0.007 \\
\hline \multicolumn{7}{|l|}{ Risk score } \\
\hline Low & 1 (reference) & & & 1 (reference) & & \\
\hline High & 64.88 & $22.55-284.86$ & $<0.001$ & 192.07 & $41.20-2087.85$ & $<0.001$ \\
\hline
\end{tabular}

FIGO, International Federation of Gynecology and Obstetrics; OR, odds ratio; inf, infinity.

\section{Discussion}

Previous studies have focused on the function of single AS events associated with ovarian cancer. Elevated expression of glutathione-specific $\gamma$-glutamylcyclotransferase splicing variants has been related to poor outcomes in ovarian cancer (35). Researchers have also found that an increased level of the mesenchymal spliced variant CD44s and reduced expression of the epithelial variant CD44v promotes epithelial-mesenchymal transition and invasion of ovarian cancer cells (36). A splice variant of the tetraspanin KAI1 mitigates its tumor-suppressive function, inducing cell migration and resulting in poor prognosis (37). Chemotherapy sensitivity is the main factor influencing survival in serous ovarian cancer (38). However, to the best of our knowledge, only a few studies have investigated the potential role of AS events in chemotherapy resistance of ovarian cancer $(39,40)$. AS events of the multidrug resistance-associated protein 1 gene in ovarian tumors have been reported to confer resistance to doxorubicin therapy (39). Overexpression of the VIII-deficient excision repair cross-complementing group 1 (ERCC1) exon is able to enhance cisplatin sensitivity in ovarian cancer cell lines by reducing the protein expression levels of ERCC1 (40). The present study demonstrated that the ES event of the ERCC1 gene was a protective factor for chemotherapy resistance, with an OR of 0.069 and a 95\% CI of 0.008-0.638 (Table SI), indicating that these results are consistent with the aforementioned study. Hence, these studies demonstrated the potential role of AS in chemotherapy resistance of $\mathrm{OV}$, and further systematic studies of AS signatures in OV may help to identify potential biomarkers and targets for chemoresistance.

The present study systemically analyzed the role of AS signatures in chemotherapy resistance using data from
320 patients with OV from the TCGA database, and then built powerful resistance predictors. A total of 22,036 AS events were detected in 7,404 genes. Approximately $38 \%$ of the AS events were ES, and the risk model based on ES events exhibited high efficiency. ES events can be validated by PCR. Thus, future research should investigate associations between ES events and chemotherapy resistance in more detail. The predictive model based on all types of AS had the best efficiency, with the AUC of the ROC curve reaching 0.931. This was much higher than the AUC for models based on a single type of AS and was more efficient than previous predictors based on single mRNA expression (AUC, 0.8056) (41), the lncRNA signature (AUC,0.83) (42) or the clinical serum CA125/ascites leptin (AUC, 0.846) (43). These combined results suggest that this model could provide accurate predictions of chemotherapy resistance in patients with $\mathrm{OV}$.

Additionally, the present study investigated the potential role of splicing factors in chemotherapy resistance. Five splicing factors were associated with chemotherapy resistance, and their possible targets were identified. These results suggested that splicing factors were involved in chemotherapy resistance in patients with serous ovarian cancer. Further work is required to determine whether regulation of these specific splicing factors could increase the sensitivity to chemotherapy and prevent disease recurrence.

The present study presented some limitations. The present study was based on RNA-seq data from the TCGA database. Validation using other databases or larger cohorts is required in future studies. Numerous splicing events and splicing factors that may be associated with the biological behavior of $\mathrm{OV}$ were identified and should be further evaluated in future experimental studies. 
Table V. Information for splicing factors in the correlation network from SpliceAid 2.

\begin{tabular}{|c|c|c|c|c|}
\hline Splicing factors & $\begin{array}{c}\text { Gene } \\
\text { names }\end{array}$ & Descriptions & $\begin{array}{l}\text { Expression in } \\
\text { normal ovary tissue }\end{array}$ & $\begin{array}{l}\text { Expression in } \\
\text { ovarian cancer }\end{array}$ \\
\hline hnRNP I (PTB) & PTBP1 & $\begin{array}{l}\text { Polypyrimidine tract binding } \\
\text { protein } 1 . \text { In the context of CALCA } \\
\text { gene, PTB enhances exon } 4 \text { inclusion } \\
\text { (PMID:9858533). nPTB functionally } \\
\text { compensates for PTB and is upregulated } \\
\text { when PTB is removed (PMID:17679092). }\end{array}$ & $\mathrm{P}(3) \_\mathrm{M}(4)$ & $\mathrm{H}(1) \_\mathrm{M}(4) \_\mathrm{L}(5)$ \\
\hline HTra2beta1 & TRA2B & Splicing factor arginine/serine-rich 10 . & $\mathrm{P}(3) \_\mathrm{L}(4)$ & $\mathrm{H}(1) \_\mathrm{M}(4) \_\mathrm{L}(5)$ \\
\hline $\mathrm{HuD}$ & ELAVL4 & $\begin{array}{l}\text { Embryonic lethal abnormal vision } \\
\text { Drosophila-like } 4 \text { (Hu antigen D). }\end{array}$ & $\mathrm{A}(4)$ & $\mathrm{A}(1) \_\mathrm{L}(4) \_\mathrm{A}(5)$ \\
\hline YB-1 & YBX1 & Y box binding protein 1. & $\mathrm{P}(3) \_\mathrm{M}(4)$ & $\mathrm{H}(1) \_\mathrm{M}(4) \_\mathrm{M}(5)$ \\
\hline hnRNP Q & SYNCRIP & $\begin{array}{l}\text { Synaptotagmin binding cytoplasmic } \\
\text { RNA interacting protein. }\end{array}$ & $\mathrm{P}(3) \_\mathrm{M}(4)$ & $\mathrm{M}(1) \_\mathrm{M}(4) \_\mathrm{L}(5)$ \\
\hline
\end{tabular}

(1) Human Protein Atlas; (2) Human Protein Reference Database; (3) Human Proteinpedia; (4) Human Transcriptome Map; (5) Cancer Genome Anatomy Project. A, absent; L, low; M, medium; H, high; P, present.

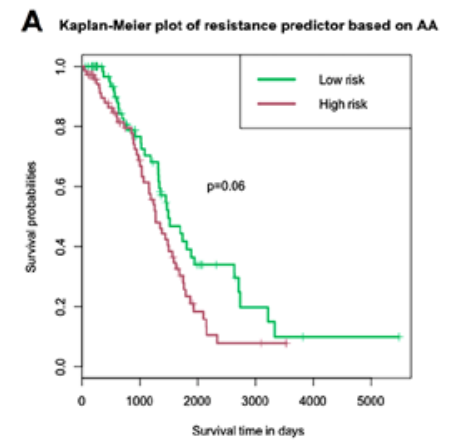

E Kaplan-Meier plot of resistance predictor based on ES

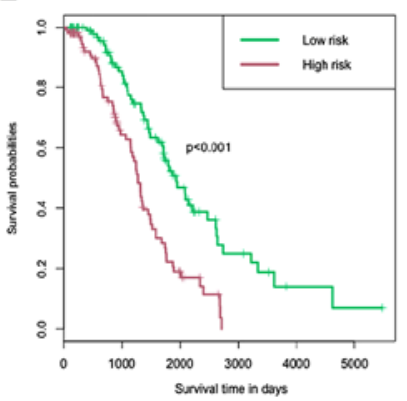

B Kaplan-Meier plot of resistance predictor based on AD

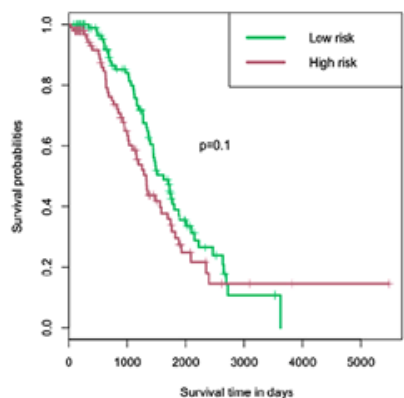

F Kaplan-Meier plot of resistance predictor based on ME

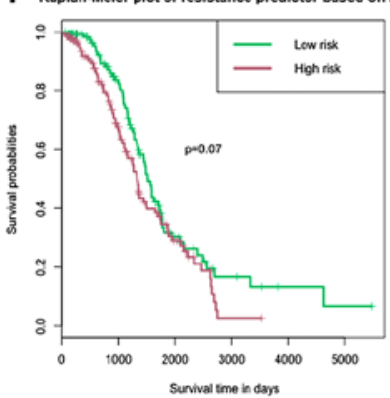

C Kaplan-Meier plot of resistance prodictor based on AP

D Kaplan-Meier plot of resistance predictor based on AT
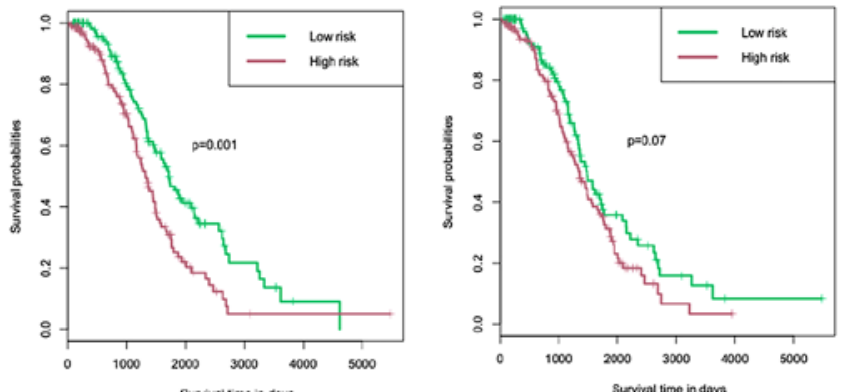

G

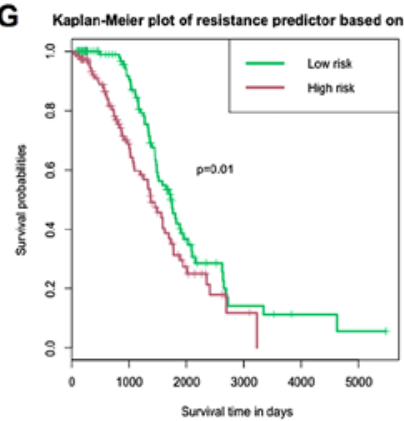

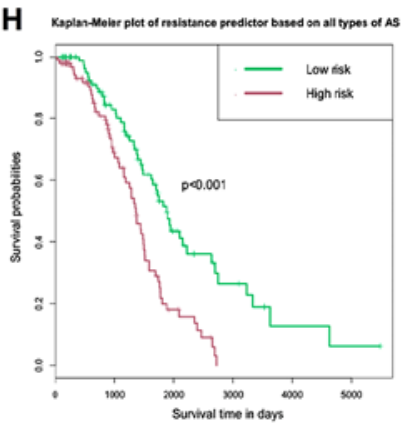

Figure 4. Kaplan-Meier plots of chemoresistance predictors for patients with OV. (A) Kaplan-Meier curves of chemoresistance predictors built with AA events for patients with OV. (B) Kaplan-Meier curves of chemoresistance predictors built with AD events for patients with OV. (C) Kaplan-Meier curves of chemoresistance predictors built with AP events for patients with OV. (D) Kaplan-Meier curves of chemoresistance predictors built with AT events for patients with OV. (E) Kaplan-Meier curves of chemoresistance predictors built with ES events for patients with OV. (F) Kaplan-Meier curves of chemoresistance predictors built with ME events for patients with OV. (G) Kaplan-Meier curves of chemoresistance predictors built with RI events for patients with OV. (H) Kaplan-Meier curves of the chemoresistance predictor built with all types of AS events for patients with OV. AS, alternative splicing; OV, ovarian serous cystadenocarcinoma; AA, alternate acceptor site; AD, alternate donor site; AP, alternate promoter; AT, alternate terminator; ES, exon skip; ME, mutually exclusive exons; RI, retained intron.

In summary, the present study determined that AS events provided valuable predictors for chemotherapy resistance. The model used provided efficient risk stratification for predicting chemotherapy resistance in patients with OV. A splicing correlation network was generated to explore the potential relationship between splicing factors and AS. A number of valuable targets were identified for future validation. The present study elucidated the role of AS events in primary platinum-based chemoresistance in patients with serous ovarian cancer and provided potential targets to overcome chemoresistance. 

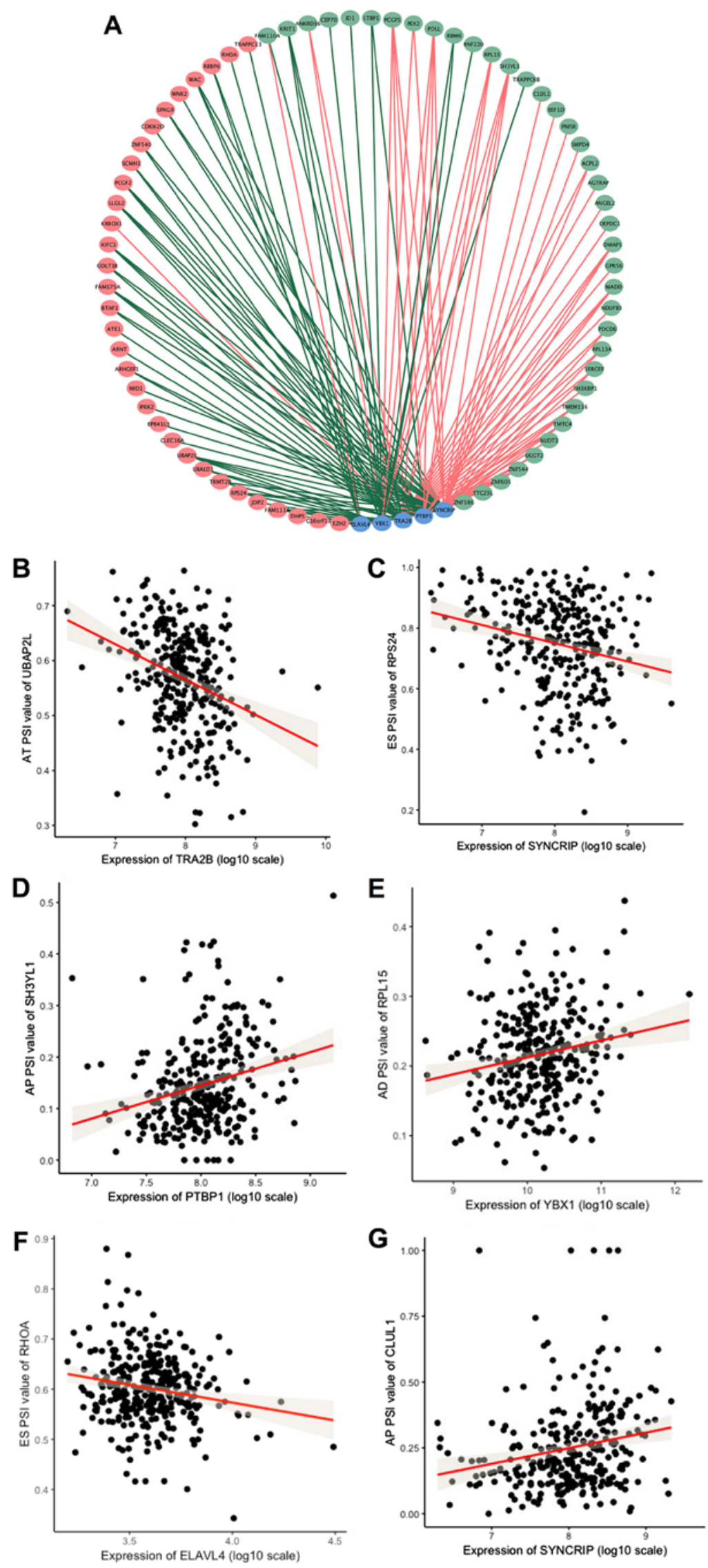

Figure 5. Splicing correlation network in OV. (A) Expression of five survival-associated splicing factors (blue circles) was positively (red lines) or negatively (green lines) correlated with the PSI values of 38 favorable (green circles) or 32 adverse prognosis AS events (red circles). (B) AT PSI value of UBAP2L was negatively correlated with expression of TRA2B. (C) ES PSI value of RPS24 was negatively correlated with expression of SYNCRIP. (D) AP PSI value of SH3YL1 was positively correlated with expression of PTBP1. (E) AD PSI value of RPL15 was positively correlated with expression of YBX1. (F) ES PSI value of RHOA was negatively correlated with expression of ELAVL4. (G) AP PSI value of CLUL1 was positively correlated with expression of SYNCRIP. P $<0.05$. AS, alternative splicing; OV, ovarian serous cystadenocarcinoma; PSI, percent spliced-in. 


\section{Acknowledgements}

Not applicable.

\section{Funding}

The present study was supported by The National Natural Science Foundation of China (grant no. 81872125) and The Research Fund for the Science and Welfare Career of Liaoning Province (grant no. 20170017).

\section{Availability of data and materials}

The datasets used and/or analyzed during the present study are available from the corresponding author on reasonable request.

\section{Authors' contributions}

TS and QY designed the study. TS performed the statistical analyses and wrote the manuscript. QY revised and edited the manuscript. All authors read and approved the final manuscript.

\section{Ethics approval and consent to participate}

In the original article of the datasets, the trials were approved by the local institutional review boards of all participating centers, and informed consent was obtained from all patients.

\section{Patient consent for publication}

Not applicable.

\section{Competing interests}

The authors declare that they have no competing interests.

\section{References}

1. Siegel RL, Miller KD and Jemal A: Cancer statistics, 2018. CA Cancer J Clin 68: 7-30, 2018.

2. Bray F, Ferlay J, Soerjomataram I, Siegel RL, Torre LA and Jemal A: Global cancer statistics 2018: GLOBOCAN estimates of incidence and mortality worldwide for 36 cancers in 185 countries. CA Cancer J Clin 68: 394-424, 2018.

3. Torre LA, Trabert B, DeSantis CE, Miller KD, Samimi G, Runowicz CD, Gaudet MM, Jemal A and Siegel RL: Ovarian cancer statistics, 2018. CA Cancer J Clin 68: 284-296, 2018.

4. Jessmon P, Boulanger T,Zhou W and Patwardhan P: Epidemiology and treatment patterns of epithelial ovarian cancer. Expert Rey Anticancer Ther 17: 427-437, 2017.

5. Bowtell DD, Böhm S, Ahmed AA, Aspuria PJ, Bast RC, Beral V, Berek JS, Birrer MJ, Blagden S, Bookman MA, et al: Rethinking ovarian cancer II: Reducing mortality from high-grade serous ovarian cancer. Nat Rev Cancer 15: 668-679, 2015.

6. Ryner L, Guan Y, Firestein R, Xiao Y, Choi Y, Rabe C, Lu S, Fuentes E, Huw LY, Lackner MR, et al: Upregulation of Periostin and reactive Stroma is associated with primary chemoresistance and predicts clinical outcomes in epithelial ovarian cancer. Clin Cancer Res 21: 2941-2951, 2015.

7. Muñoz-Galván S, Felipe-Abrio B, García-Carrasco M, Domínguez-Piñol J, Suarez-Martinez E, Verdugo-Sivianes EM, Espinosa-Sánchez A, Navas LE, Otero-Albiol D, Marin JJ, et al: New markers for human ovarian cancer that link platinum resistance to the cancer stem cell phenotype and define new therapeutic combinations and diagnostic tools. J Exp Clin Cancer Res 38: 234, 2019.
8. van Zyl B, Tang D and Bowden NA: Biomarkers of platinum resistance in ovarian cancer: What can we use to improve treatment. Endocr Relat Cancer 25: R303-R318, 2018.

9. Shu T, Li Y, Wu X, Li B and Liu Z: Down-regulation of HECTD3 by HER2 inhibition makes serous ovarian cancer cells sensitive to platinum treatment. Cancer Lett 411: 65-73, 2017.

10. Bai L, Wang A, Zhang Y, Xu X and Zhang X: Knockdown of MALAT1 enhances chemosensitivity of ovarian cancer cells to cisplatin through inhibiting the Notch1 signaling pathway. Exp Cell Res 366: 161-171, 2018.

11. Bai H, Cao D, Yang J, Li M, Zhang Z and Shen K: Genetic and epigenetic heterogeneity of epithelial ovarian cancer and the clinical implications for molecular targeted therapy. J Cell Mol Med 20: 581-593, 2016.

12. Konecny GE, Winterhoff B and Wang C: Gene-expression signatures in ovarian cancer: Promise and challenges for patient stratification. Gynecol Oncol 141: 379-385, 2016.

13. Chon HS and Lancaster JM: Microarray-based gene expression studies in ovarian cancer. Cancer Control 18: 8-15, 2011.

14. Salton M and Misteli T: Small molecule modulators of Pre-mRNA splicing in cancer therapy. Trends Mol Med 22: 28-37, 2016.

15. Narayanan SP, Singh S and Shukla S: A saga of cancer epigenetics: Linking epigenetics to alternative splicing. Biochem J 474: 885-896, 2017.

16. Nilsen TW and Graveley BR: Expansion of the eukaryotic proteome by alternative splicing. Nature 463: 457-463, 2010.

17. Nakka K, Ghigna C, Gabellini D and Dilworth FJ: Diversification of the muscle proteome through alternative splicing. Skelet Muscle 8: 8, 2018.

18. Chang SH, Elemento O, Zhang J, Zhuang ZW, Simons M and Hla T: ELAVL1 regulates alternative splicing of eIF4E transporter to promote postnatal angiogenesis. Proc Natl Acad Sci USA 111: 18309-18314, 2014.

19. Wang Y, Liu Y, Nie H, Ma X and Xu Z: Alternative splicing of inner-ear-expressed genes. Front Med 10: 250-257, 2016.

20. Lin L, Park JW, Ramachandran S, Zhang Y, Tseng YT, Shen S, Waldvogel HJ, Curtis MA, Faull RL, Troncoso JC, et al: Transcriptome sequencing reveals aberrant alternative splicing in Huntington's disease. Hum Mol Genet 25: 3454-3466, 2016.

21. Urbanski LM, Leclair N and Anczuków O: Alternative-splicing defects in cancer: Splicing regulators and their downstream targets, guiding the way to novel cancer therapeutics. Wiley Interdiscip Rev RNA 9: e1476, 2018.

22. Martinez-Montiel N, Rosas-Murrieta NH, Anaya Ruiz M, Monjaraz-Guzman E and Martinez-Contreras R: Alternative splicing as a target for cancer treatment. Int J Mol Sci 19: pii: E545, 2018 .

23. Dvinge H, Kim E, Abdel-Wahab O and Bradley RK: RNA splicing factors as oncoproteins and tumour suppressors. Nat Rev Cancer 16: 413-430, 2016.

24. Sveen A, Kilpinen S, Ruusulehto A, Lothe RA and Skotheim RI: Aberrant RNA splicing in cancer; Expression changes and driver mutations of splicing factor genes. Oncogene 35: 2413-2427, 2016.

25. Shen S, Wang Y, Wang C, Wu YN and Xing Y: SURVIV for survival analysis of mRNA isoform variation. Nat Commun 7: 11548, 2016.

26. Song J, Liu YD, Su J, Yuan D, Sun F and Zhu J: Systematic analysis of alternative splicing signature unveils prognostic predictor for kidney renal clear cell carcinoma. J Cell Physiol 234: 22753-22764, 2019.

27. Zhu GQ, Zhou YJ, Qiu LX, Wang B, Yang Y, Liao WT, Luo YH, Shi YH, Zhou J, Fan J and Dai Z: Prognostic alternative mRNA splicing signature in hepatocellular carcinoma: A study based on large-scale sequencing data. Carcinogenesis: May 17, 2019 doi: 10.1093/carcin/bgz073 (Epub ahead of print).

28. Zhu J, Chen Z and Yong L: Systematic profiling of alternative splicing signature reveals prognostic predictor for ovarian cancer. Gynecol Oncol 148: 368-374, 2018.

29. Ryan M, Wong WC, Brown R, Akbani R, Su X, Broom B, Melott J and Weinstein J: TCGASpliceSeq a compendium of alternative mRNA splicing in cancer. Nucleic Acids Res 44: D1018-D1022, 2016.

30. Berger AC, Korkut A, Kanchi RS, Hegde AM, Lenoir W, Liu W, Liu Y, Fan H, Shen H, Ravikumar V, et al: A Comprehensive Pan-cancer molecular study of gynecologic and breast cancers. Cancer Cell 33: 690-705.e9, 2018.

31. Piva F, Giulietti M, Burini AB and Principato G: SpliceAid 2: A database of human splicing factors expression data and RNA target motifs. Hum Mutat 33: 81-85, 2012. 
32. Akaike H: Information theory and an extension of the maximum likelihood principle. 2nd Int. Sympo. on Information Theor, 1972. https://doi.org/10.1007/978-1-4612-0919-5 38

33. Jayson GC, Kohn EC, Kitchener HC and Ledermann JA: Ovarian cancer. Lancet 384: 1376-1388, 2014.

34. Dennis G Jr, Sherman BT, Hosack DA, Yang J, Gao W, Lane HC and Lempicki RA: DAVID: Database for annotation, visualization, and integrated discovery. Genome Biol 4: P3, 2003.

35. Goebel G, Berger R, Strasak AM, Egle D, Müller-Holzner E, Schmidt S, Rainer J, Presul E, Parson W, Lang S, et al: Elevated mRNA expression of CHAC1 splicing variants is associated with poor outcome for breast and ovarian cancer patients. Br J Cancer 106: 189-198, 2012.

36. Bhattacharya R, Mitra T, Ray Chaudhuri S and Roy SS: Mesenchymal splice isoform of CD44 (CD44s) promotes EMT/invasion and imparts stem-like properties to ovarian cancer cells. J Cell Biochem 119: 3373-3383, 2018.

37. Upheber S, Karle A, Miller J, Schlaugk S, Gross E and Reuning U: Alternative splicing of KAI1 abrogates its tumor-suppressive effects on integrin $\alpha v \beta 3$-mediated ovarian cancer biology. Cell Signal 27: 652-662, 2015.

38. Markman M: Antineoplastic agents in the management of ovarian cancer: Current status and emerging therapeutic strategies. Trends Pharmacol Sci 29: 515-519, 2018.
39. He X, Ee PL, Coon JS and Beck WT: Alternative splicing of the multidrug resistance protein 1/ATP binding cassette transporter subfamily gene in ovarian cancer creates functional splice variants and is associated with increased expression of the splicing factors PTB and SRp20. Clin Cancer Res 10: 4652-4660, 2004.

40. Sun Y, Li T, Ma K, Tian Z, Zhu Y, Chen F and Hu G: The impacts of ERCC1 gene exon VIII alternative splicing on cisplatin-resistance in ovarian cancer cells. Cancer Invest 27: 891-897, 2009

41. Zhao H, Sun Q, Li L, Zhou J, Zhang C, Hu T, Zhou X, Zhang L, Wang B, Li B, et al: High expression levels of AGGF1 and MFAP4 predict primary platinum-based chemoresistance and are associated with adverse prognosis in patients with serous ovarian cancer. J Cancer 10: 397-407, 2019.

42. Liu R, Zeng Y, Zhou CF, Wang Y, Li X, Liu ZQ, Chen XP, Zhang $\mathrm{W}$ and Zhou HH: Long noncoding RNA expression signature to predict platinum-based chemotherapeutic sensitivity of ovarian cancer patients. Sci Rep 7: 18, 2017.

43. Matte I, Garde-Granger P, Bessette P and Piché A: Serum CA125 and ascites leptin level ratio predicts baseline clinical resistance to first-line platinum-based treatment and poor prognosis in patients with high grade serous ovarian cancer. Am J Cancer Res 9: 160-170, 2019. 